\title{
Frequency-Based Separation of Climate Signals
}

\author{
Alexander Ilin ${ }^{1}$ and Harri Valpola ${ }^{2}$ \\ 1 Helsinki University of Technology, Neural Networks Research Centre, \\ P.O. Box 5400, FI-02015 TKK, Espoo, Finland \\ Alexander.Ilin@tkk.fi \\ 2 Helsinki University of Technology, Lab. of Computational Engineering, \\ P.O. Box 9203, FI-02015 TKK, Espoo, Finland \\ Harri.Valpola@tkk.fi \\ http://www.cis.hut.fi/projects/dss/
}

\begin{abstract}
The paper presents an example of exploratory data analysis of climate measurements using a recently developed denoising source separation (DSS) framework. We analysed a combined dataset containing daily measurements of three variables: surface temperature, sea level pressure and precipitation around the globe. Components exhibiting slow temporal behaviour were extracted using DSS with linear denoising. These slow components were further rotated using DSS with nonlinear denoising which implemented a frequency-based separation criterion. The rotated sources give a meaningful representation of the slow climate variability as a combination of trends, interannual oscillations, the annual cycle and slowly changing seasonal variations.
\end{abstract}

\section{Introduction}

One of the main goals of statistical analysis of climate data is to extract physically meaningful patterns of climate variability from highly multivariate weather measurements. The classical technique for defining such dominant patterns is principal component analysis (PCA) or empirical orthogonal functions (EOF) as it is called in climatology (see, e.g., [1]). However, many researchers pointed out that the maximum remaining variance criterion used in PCA can lead to such problems as mixing different physical phenomena in one extracted component [2 3]. This makes PCA a useful tool for information compression but limits its ability to isolate individual modes of climate variation.

To overcome this problem, rotation of the principal components proved useful [2]. The different rotation criteria reviewed in [2] are based on the general "simple structure" idea aimed at, for example, spatial or temporal localisation of the rotated components. The rotation of EOFs can be either orthogonal or oblique, which potentially leads to better interpretability of the extracted components.

Independent component analysis (ICA) is a recently developed statistical technique for component extraction which can also be used for rotating principal components. The basic assumption made in ICA is the statistical independence of the extracted components, which may lead to a meaningful data representation in 
a number of applications (see, e.g., 4 for introduction). ICA is based on higherorder statistics and in this respect bears some similarity to classical rotation techniques such as the Varimax orthogonal rotation [2]. Several attempts to apply ICA in climate research have already been made [56].

In this paper, we analyse weather measurements using a novel extension of ICA called denoising source separation 7]. DSS is a general separation framework which does not necessarily exploit the independence assumption but rather looks for hidden components which have "interesting" properties. The interestingness of the properties is controlled by means of a denoising procedure. For example, in [8], the sources with most prominent interannual oscillations were identified using DSS with linear filtering as denoising. The leading components were clearly related to the well-known El Niño-Southern Oscillation (ENSO) phenomenon and several other interesting components were extracted as well.

In the present work, we use DSS with linear denoising as the first, preprocessing step of climate data analysis. A wider frequency band in the denoising filter is used to identify the slow subspace of the climate system. The found slow components are further rotated using an iterative DSS procedure based on nonlinear denoising. The rotation is done such that the extracted components would have distinct power spectra.

The extracted components turned out to represent the subspace of the slow climate phenomena as a linear combination of trends, decadal-interannual oscillations, the annual cycle and other phenomena with distinct spectral contents. Using this approach, the known climate phenomena are identified as certain subspaces of the climate system and some other interesting phenomena hidden in the weather measurements are found.

\section{DSS Method}

DSS is a general algorithmic framework which can be used for discovering interesting phenomena hidden in multivariate data 7. Similarly to PCA, ICA or other rotation techniques, DSS is based on the linear mixing model. The basic assumption is that there are some hidden components $\mathbf{s}(t)$ (also called sources or factors) which are reflected in the measurements $\mathbf{x}(t)$ through a linear mapping: $\mathbf{x}(t)=\mathbf{A s}(t)$. The mapping $\mathbf{A}$ is called the mixing matrix in the ICA terminology or the loading matrix in the context of PCA.

The goal of the analysis is to estimate the unknown components $\mathbf{s}(t)$ and the corresponding loading vectors (the columns of $\mathbf{A}$ ) from the observed data $\mathbf{x}(t)$. In the climate data analysis, the components usually correspond to the timevarying states of the climate system and the loading vectors are the spatial maps showing the typical weather patterns corresponding to the found components. The components $\mathbf{s}(t)$ are usually normalised to unit variances, and therefore the spatial patterns have a meaningful scale.

The first step of DSS is so-called whitening or sphering (see Fig. 1). The goal of whitening is to uniform the covariance structure of the data in such a way that any linear projection of the data has unit variance. The positive 


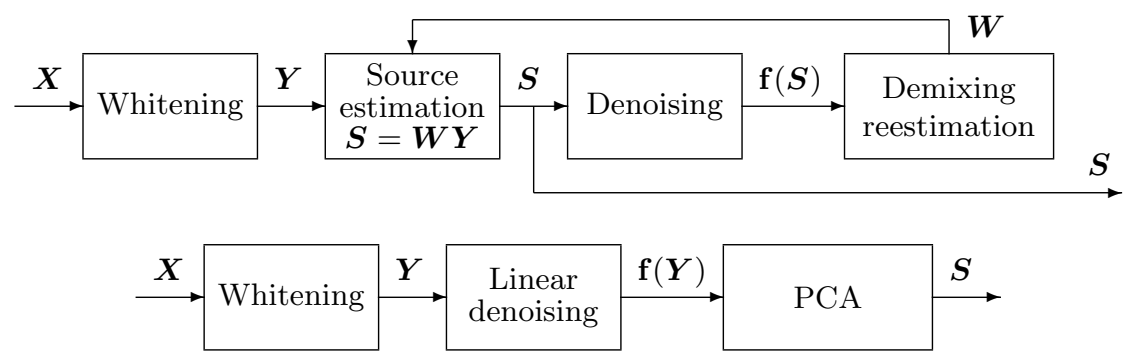

Fig. 1. The steps of the DSS algorithm in the general case (above) and in the case of linear denoising (below)

effect of such transformation is that any orthogonal basis in the whitened space defines uncorrelated sources. Therefore, whitening is used as a preprocessing step in many ICA algorithms, which allows restricting the mixing matrix to be orthogonal afterwards. Whitening is usually implemented by PCA.

The following stage is an orthogonal rotation of the white components $\boldsymbol{Y}$ based on the source separation criterion defined in the form of a denoising procedure. It is in general done using an iterative algorithm with three steps: source estimation, denoising of the source estimates and reestimation of the demixing matrix. Without denoising, this procedure is equivalent to the power method for computing the principal component of $\boldsymbol{Y}$. Since $\boldsymbol{Y}$ is white, all the eigenvalues are equal and the solution without denoising becomes degenerate. Therefore, even slightest changes made by denoising can determine the DSS rotation. Since the denoising procedure emphasises the desired properties of the sources, DSS can find the rotation where the properties of interest are maximised.

Linear denoising is a simpler case as it does not require the described iterative procedure. DSS based on linear denoising can be performed in three steps: whitening, denoising and PCA on the denoised data (see Fig. 1). The idea behind this approach is that denoising renders the variances of the sphered components different and PCA can identify the directions which maximise the properties of interest. The eigenvalues given by PCA tell the ratio of the variance of the sources after and before filtering which is the objective function in linear denoising. The components are ranked according to the prominence of the desired properties the same way as the principal components in PCA are ranked according to the amount of variance they explain.

More general nonlinear denoising can implement more complicated separation criteria (see [79] for several examples). The objective function is usually expressed implicitly in the denoising function. Therefore, ranking the components is more difficult in this case and depends on the exact separation criterion used in the denoising procedure.

In the present work, DSS is exploited twice. First, DSS with linear denoising extracts components which exhibit most prominent variability in the slow time scale. Therefore, linear denoising is implemented using a low-pass filter whose frequency response is shown in Fig. 2. A similar approach (but with another 


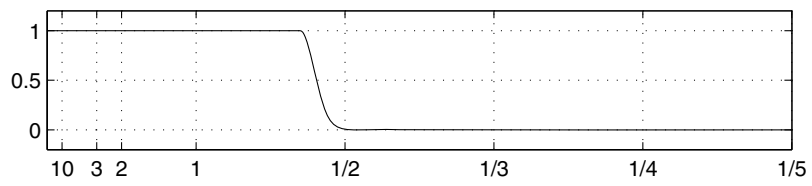

Fig. 2. Frequency response of the filter used in DSS with linear denoising. The abscissa is linear in frequency but is labeled in terms of periods, in years.

type of filter) was introduced in [8] to identify the subspace with most prominent interannual variability.

After that, the slow components are rotated such that they would have distinct power spectra. This is done by DSS with the general iterative procedure in which denoising implements a frequency-based separation criterion. Practically, the denoising procedure is based on whitening smoothed discrete cosine transform (DCT) power spectra of the components and using inverse DCT to calculate the denoised sources $\mathbf{f}(\boldsymbol{S})$. This denoising mechanism is somewhat similar to the whitening-based estimation of the source variance proposed in [9]. The algorithm also tries to order the sources according to their frequencies using topographic ideas somewhat similar to [10].

\section{Data and Preprocessing Method}

The proposed technique is applied to measurements of three major atmospheric variables: surface temperature, sea level pressure and precipitation. This set of variables is often used for describing global climate phenomena such as ENSO [11. The datasets are provided by the reanalysis project of the National Centers for Environmental Prediction-National Center for Atmospheric Research (NCEP/NCAR) [12,1]

The data represent globally gridded measurements over a long period of time. The spatial grid is regularly spaced over the globe with $2.5^{\circ} \times 2.5^{\circ}$ resolution. Although the quality of the data is worse for the beginning of the reanalysis period and it considerably varies throughout the globe, we used the whole period of $1948-2004$.

The long-term mean was removed from the data and the data points were weighed similarly to 8 to diminish the effect of a denser sampling grid around the poles. Each data point was multiplied by a weight proportional to the square root of the corresponding area of its location. The spatial dimensionality of the data was reduced using the PCA/EOF analysis applied to the weighed data. For each dataset, we retained 100 principal components which explain more than $90 \%$ of the total variance. The DSS analysis was then applied to the combined data containing the measurements of the three variables.

${ }^{1}$ The authors would like to thank the NOAA-CIRES Climate Diagnostics Center, Boulder, Colorado, USA, for providing NCEP Reanalysis data from their Web site at http://www.cdc.noaa.gov. 


\section{Results}

First, we identified the subspace of slow climate phenomena by applying DSS with low-pass filtering as linear denoising to the daily weather measurements. The time course of the most prominent slow components extracted from highly multidimensional data is shown in the leftmost column of Fig. 3. The annual cycle appears in the two leading components as the clearest slow source of the climate variability. The following components also possess interesting slow behaviour.

However, the sources found at this stage appear to be mixtures of several climate phenomena. For example, the third and the fourth components are mixtures of slow trends and the prominent ENSO oscillations. Similar mixed phenomena can be found in other components as well. This effect is also seen from the power spectra of the components (not shown here). Many components possess very prominent slowest, decadal or close-to-annual frequencies. Except for the two annual cycle sources, none of the components has a clear dominant peak in its power spectrum.

The first sixteen slow components extracted by DSS with linear denoising were further rotated using frequency-based DSS described in Section 2, To discard high-frequency noise, the monthly averages of the slow components were used at this stage. The time course of the rotated sources is presented in Fig. 3 and the spatial patterns corresponding to some of the sources are shown in Fig. 4 . The components now have more clear interpretation compared to the original slow components.

The power spectra of the rotated components are more distinct (see the rightmost column of Fig. 3). However, some of the power spectra look quite similar and we can roughly categorise the found sources into three subspaces with different variability time scales: trends (components 1-5), interannual oscillations (components 6-11) and components 12-16 with dominating close-to-annual frequencies in their spectra. The subspaces are identified reliably due to the distinct differences in the corresponding power spectra but the components within the subspaces may remain mixed.

Among the slowest climate trends, the most prominent one is component 3 which has a constantly increasing time course. This component may be related to global warming as the corresponding surface temperature map has mostly positive values all over the globe (see Fig. 4). The highest temperature loadings of this component are mainly concentrated around the North and South Poles, the sea level pressure map has a clear localisation around the South Pole and the precipitation loadings are mostly located in the tropical regions.

Components 6-11 exhibit prominent oscillatory behaviour in the interannual time scale. The most prominent sources here are components 7 and 8 which are closely related to the ENSO oscillations both in the time course and in the corresponding spatial patterns (see Fig. 4). They are very similar to the first two components extracted in [8]: component 7 is similar to the ENSO index and component 8 bears resemblance with the differential ENSO index. Component 6 may be related to the slower behaviour of ENSO. Component 10 has very distinct spatial patterns with a prominent dipole structure over the continents 
Slow Subspace

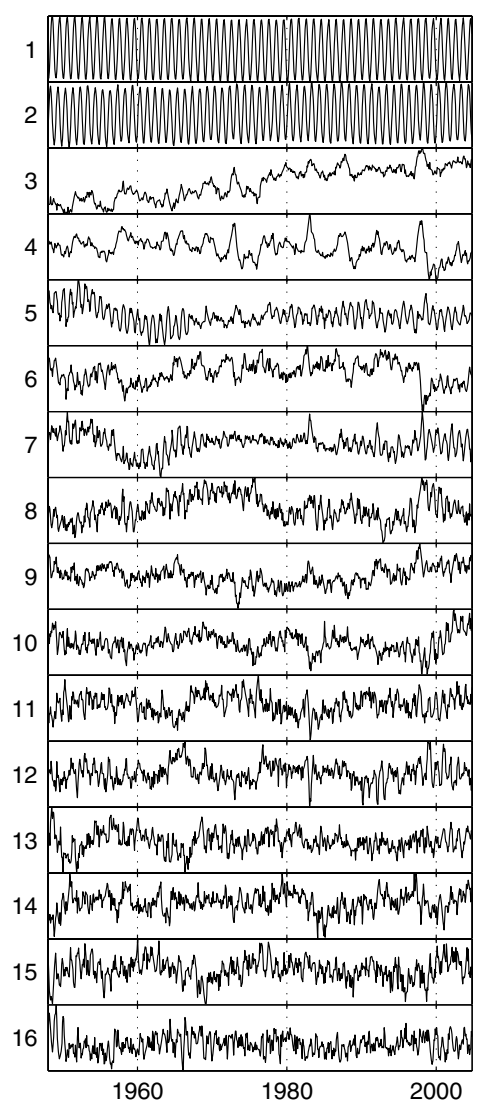

Frequency-Based Separation

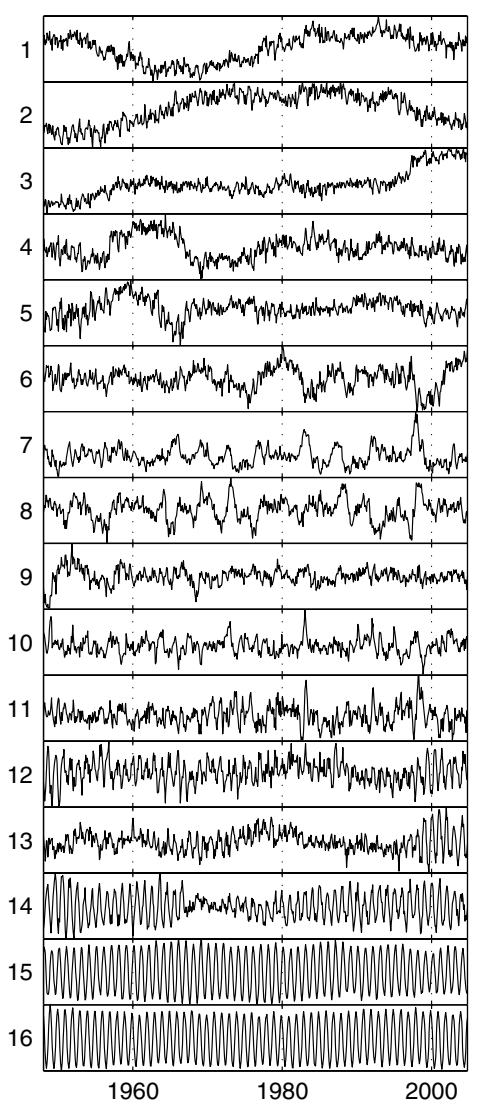

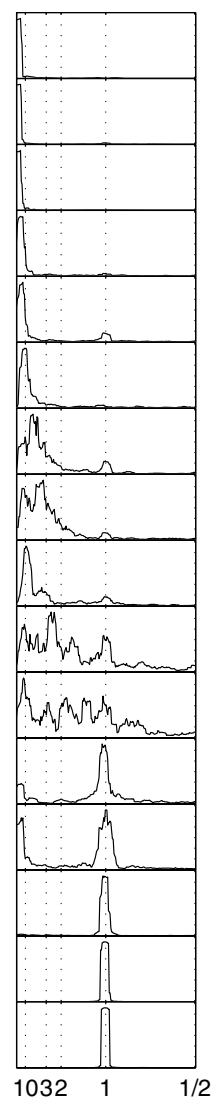

Fig. 3. Left: The monthly averages of the components extracted by DSS with linear denoising. Middle: The rotated slow components estimated by frequency-based DSS. The variances of all the components are normalised to unity. Right: The power spectra of the components found by frequency-based DSS. The abscissa is linear in frequency but is labeled in terms of periods, in years.

in the Northern Hemisphere in the temperature maps. A similar source was also extracted in 8].

Components 12-16 have prominent close-to-annual frequencies in their power spectra. The annual cycle now appears in components 15-16. The rest of the sources resemble the annual oscillations modulated (multipiled) by some slow signals. Thus, this set of components may be related to some phenomena slowly changing the annual cycle. However, as we already pointed out, the found rotation within this subspace may not be most meaningful. 
Surface temperature, ${ }^{\circ} \mathrm{C} \quad$ Sea level pressure, $\mathrm{Pa} \quad$ Precipitation, $\mathrm{kg} / \mathrm{m}^{2}$
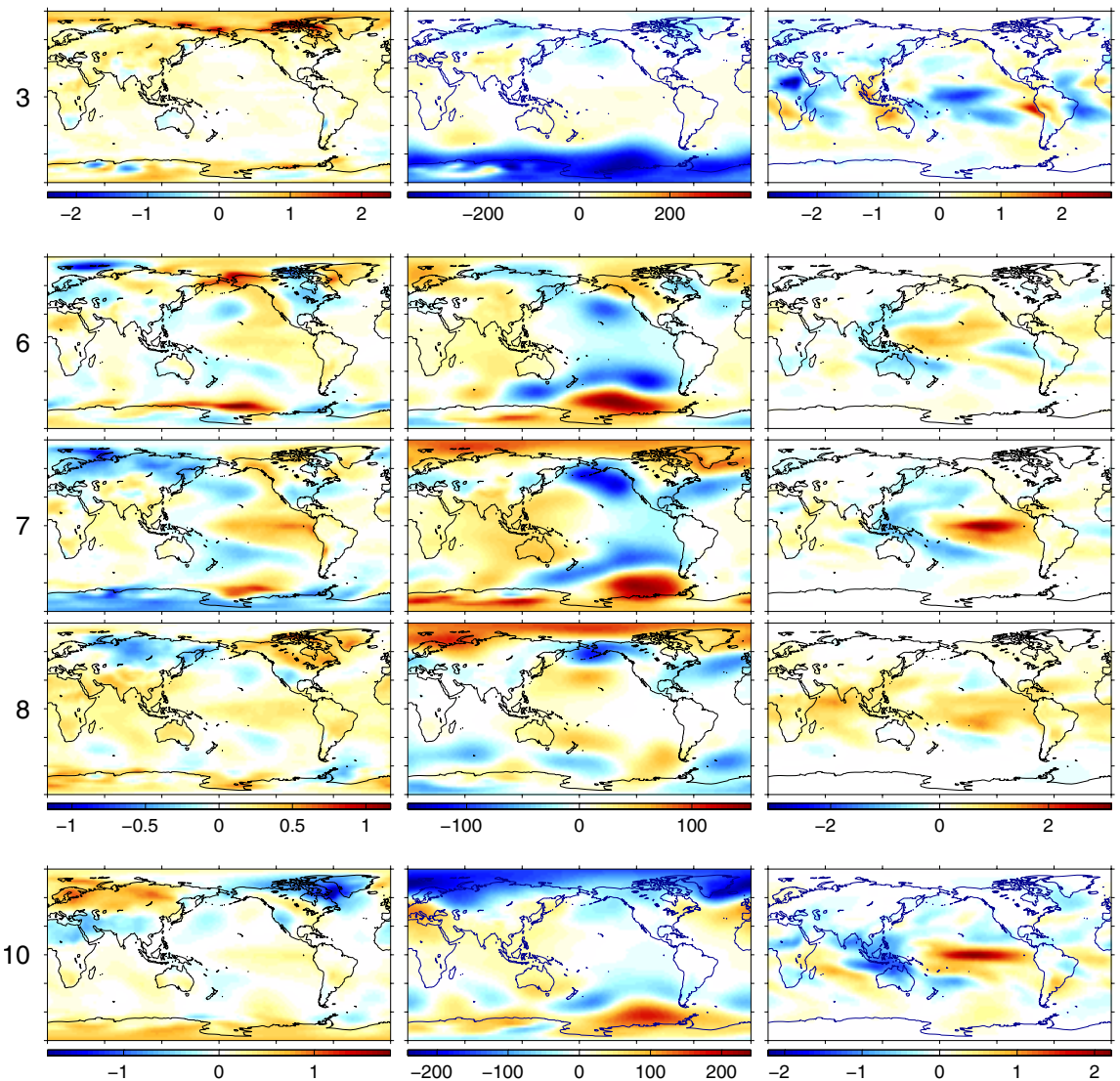

Fig. 4. The spatial patterns of several components found by frequency-based DSS. The label on the left indicates the number of the component in Fig. 3

\section{Discussion}

In this paper, we showed how the DSS framework can be applied to exploratory analysis of climate data. We used a frequency-based separation criterion to identify slow varying climate phenomena with distinct temporal behaviour. The presented algorithm can be used for both finding a physically meaningful representation of the data and for an easier interpretation of the complex climate variability. It can also be useful for making predictions of future measurements or for detecting artifacts produced during the data acquisition.

Representing climate variability as a combination of hidden phenomena does not have a unique solution because of the high complexity of the climate system where different phenomena constantly interact with each other. This task always allows some subjectivity where the exact details of the separation procedure de- 
pend on the ultimate goal of research. A good example of such subjectivity is choosing the number of extracted components in the proposed DSS procedure. In the presented experiments, we chose this number such that the components would easily be interpretable. According to our experience, increasing the number of components usually results in describing one phenomenon by several components having slightly different frequency contents. This may be useful for better understanding of well-known climate phenomena or for discovering new, not easily observable phenomena, but it may also be counter-productive if the solution becomes overfitted.

Note also that the proposed method may sometimes identify reliably only the subspaces of components having similar power spectra and the rotation within the subspaces may not be most meaningful. Some other separation criteria based on, for instance, dynamical modelling or the interaction with the seasonal variations is an important line of future research.

\section{References}

1. H. von Storch and W. Zwiers, Statistical Analysis in Climate Research. Cambridge, U.K.: Cambridge Univ. Press, 1999.

2. M. B. Richman, Rotation of principal components, J. of Climatology, vol. 6, pp. 293-335, 1986.

3. K.-Y. Kim and Q. Wu, A comparison study of EOF techniques: Analysis of nonstationary data with periodic statistics, J. of Climate, vol. 12, pp. 185-199, 1999.

4. A. Hyvärinen, J. Karhunen, and E. Oja, Independent Component Analysis. J. Wiley, 2001.

5. F. Aires, A. Chédin, and J.-P. Nadal, Independent component analysis of multivariate time series: Application to the tropical SST variability, J. of Geophysical Research, vol. 105, pp. 17, 437-17, 455, July 2000.

6. A. Lotsch, M. A. Friedl, and J. Pinzón, Spatio-temporal deconvolution of NDVI image sequences using independent component analysis, IEEE Trans. on Geoscience and Remote Sensing, vol. 41, pp. 2938-2942, December 2003.

7. J. Särelä and H. Valpola, Denoising source separation, Journal of Machine Learning Research, vol. 6, pp. 233-272, 2005.

8. A. Ilin, H. Valpola, and E. Oja, Semiblind source separation of climate data detects El Niño as the component with the highest interannual variability, in Proc. of Int. Joint Conf. on Neural Networks (IJCNN2005), (Montreal, Quebec, Canada), 2005. Accepted.

9. H. Valpola and J. Särelä, Accurate, fast and stable denoising source separation algorithms, in Proc. of Fifth Int. Conf. on Independent Component Analysis and Blind Signal Separation (ICA 2004) (C. G. Puntonet and A. Prieto, eds.), vol. 3195 of Lecture Notes in Computer Science, (Granada, Spain), pp. 65-72, SpringerVerlag, Berlin, 2004.

10. A. Hyvärinen, P. Hoyer, and M. Inki, Topographic independent component analysis, Neural Computation, vol. 13, no. 7, pp. 1525-1558, 2001.

11. K. E. Trenberth and J. M. Caron, The Southern Oscillation revisited: Sea level pressures, surface temperatures, and precipitation, Journal of Climate, vol. 13, pp. 4358-4365, December 2000.

12. E. Kalnay and Coauthors, The NCEP/NCAR 40-year reanalysis project, Bulletin of the American Meteorological Society, vol. 77, pp. 437-471, 1996. 